MARTHA NAST ${ }^{1}$

ANDRESSA DE OLIVEIRA ${ }^{1}$

FERNANDA RAUBER ${ }^{2}$

MÁrCia ReGina Vitolo ${ }^{3}$

\title{
Ganho de peso excessivo na gestação é fator de risco para o excesso de peso em mulheres
}

\author{
Excessive gestational weight gain is risk factor \\ for overweight among women
}

Artigo Original

Palavras-chave
Índice de massa corporal
Peso corporal
Obesidade

Ganho de peso/complicações Complicações na gravidez/fisiopatologia

Saúde da mulher

Fatores de risco

Keywords

Body mass index

Body weight

Obesity

Weight gain/complications

Pregnancy complications/physiopathology

Women's health

Risk factors

\section{Resumo}

OBJETIVO: Avaliar a retenção de peso 12 meses após o parto e seus fatores associados entre mulheres que realizaram o pré-natal em Unidades de Saúde da cidade de Porto Alegre. MÉTODOS: Gestantes no terceiro trimestre gestacional foram identificadas em 20 Unidades de Saúde, e dados socioeconômicos, demográficos e antropométricos foram coletados. Seis e 12 meses após o parto, realizou-se visita domiciliar às mulheres participantes para obtenção das medidas antropométricas. $\bigcirc$ ganho de peso gestacional foi avaliado considerando-se o índice de massa corporal (IMC) pré-gestacional. A retenção de peso foi obtida pela subtração do peso pré-gestacional, e o peso aferido 6 e 12 meses após o parto. Para análise dos dados, utilizou-se o Teste de McNemar, a ANOVA com as comparações múltiplas de Bonferroni e a regressão linear múltipla. RESULTADOS: Das 715 gestantes entrevistadas, 545 foram avaliadas 12 meses após o parto. A prevalência de excesso de peso 12 meses após o parto foi superior comparado ao período pré-gestacional $(52,9$ versus 36,7\%) e 30,7\% das mulhere retiveram $\geq 10 \mathrm{~kg}$. A retenção de peso 12 meses após o parto foi superior nas mulheres que apresentavam sobrepeso pré-gestacional $(9,9 \pm 7,7 \mathrm{~kg})$ em comparação àquelas eutróficas $(7,6 \pm 6,2 \mathrm{~kg})$. Maior IMC pré-gestacional, maior ganho de peso gestacional e ser adolescente foram associados com maior retenção de peso 12 meses após o parto $(p<0,001)$. CONCLUSÃO: É necessária a adequada assistência pré-natal para minimizar os efeitos adversos do ganho de peso excessivo durante a gestação na saúde da mulher.

\section{Abstract}

PURPOSE: To evaluate weight retention 12 months postpartum and factors associated among women who had received prenatal care at Health Care Centers in Porto Alegre, southern Brazil. METHODS: Pregnant women in the last trimester were identified at 20 Health Care Centers. Socioeconomic, demographic and anthropometrics data were obtained. Six and 12 months after delivery, the women received home visits for anthropometric measures. The gestational weight gain was defined by pre-pregnancy Body Mass Index (BMI). Weight retention was defined as the difference between pre-gestational weight and weight at postpartum. Data were analyzed using McNemar's Test, ANOVA with Bonferroni correction and multiple linear regression. RESULTS: Of the 715 pregnant women recruited, 545 were assessed 12 months after delivery. Women were more likely to be overweight 12 months postpartum compared to the pre-pregnancy period $(52.9$ versus $36.7 \%)$ and weight retention during the 12 months postpartum was more than $10 \mathrm{~kg}$ in $30.7 \%$ of the women. Weight retention in the postpartum period was higher among women who were overweight $(9.9 \pm 7.7 \mathrm{~kg})$ compared to those who were of normal weight during the pre-pregnancy period $(7.6 \pm 6.2 \mathrm{~kg})$. Pre-pregnancy BMI, gestational weight gain, and maternal age were associated with gestational weight retention 12 months postpartum $(p<0.001)$. CONCLUSION: Adequate prenatal care is necessary to minimize the adverse effects of excessive weight gain during pregnancy on women's health.
Correspondência

Márcia Regina Vitolo

Departamento de Nutriçāo, Universidade Federal de Ciêncios da Saúde de Porto Alegre Rua Sarmento Leite, 245 - Centro (EP: $90050-170$ Porto Alegre (RS), Brasi

Recebido

$08 / 10 / 2013$

Aceito com modificacōes $28 / 11 / 2013$
Universidade Federal de Ciências da Saúde de Porto Alegre - UFCSPA - Porto Alegre (RS), Brasil.

'Núcleo de Pesquisa em Nutrição, Universidade Federal de Ciências da Saúde de Porto Alegre - UFCSPA - Porto Alegre (RS), Brasil. 2Programa de Pós-Graduação em Ciências da Saúde, Universidade Federal de Ciências da Saúde de Porto Alegre - UFCSPA Porto Alegre (RS), Brasil.

${ }^{3}$ Departamento de Nutrição, Universidade Federal de Ciências da Saúde de Porto Alegre - UFCSPA - Porto Alegre (RS), Brasil. Conflito de interesses: nada a declarar. 
Introdução

A gravidez e o pós-parto caracterizam-se por período de risco para o desenvolvimento de excesso de peso em mulheres ${ }^{1-4}$. Durante a gestação, muitas mulheres ganham mais peso do que o recomendado 5 , o que pode alterar substanciamente seu estado nutricional futuro ${ }^{6}$. Embora a relação da gestação e risco de obesidade tenha sido foco de diversos estudos, os resultados ainda são controversos e limitados para a população de mulheres brasileiras ${ }^{7}$.

No Brasil, estudos mostraram prevalências de até $52 \%$ de mulheres com ganho de peso gestacional excessivo $^{8-10}$. Uma revisão sistemática mostrou que, além das consequências como o excesso de peso ao nascer e macrossomia infantil, o ganho de peso gestacional superior ao recomendado está associado à retenção de peso materno, definida como a diferença entre o peso pré-gestacional e o peso em determinado momento após o parto ${ }^{5}$, no curto, médio e longo prazo ${ }^{11}$. Estudos prospectivos mostraram que mais de $65 \%$ das mulheres não retornam ao peso prégestacional em até um ano após o parto ${ }^{12,13}$. No Brasil, dados de duas coortes de mulheres mostraram que $30 \%$ apresentaram valores de retenção de peso 2 meses após o parto em torno de $5 \mathrm{~kg}^{14}$ e $19,2 \%$ retiveram $7,5 \mathrm{~kg}$ ou mais 9 meses após o parto ${ }^{6}$. Outros fatores como o índice de massa corpórea pré-gestacional e características socioeconômicas também têm sido associados à retenção de peso após o parto ${ }^{15}$; contudo, ainda há uma grande lacuna na discussão dos seus determinantes.

O objetivo deste estudo foi avaliar a retenção de peso 6 e 12 meses após o parto e seus fatores associados entre mulheres que realizaram o pré-natal em Unidades de Saúde da cidade de Porto Alegre.

\section{Métodos}

Análise de dados de mulheres que participaram de um ensaio de campo randomizado por conglomerados realizado no período de abril de 2008 a março de 2010, com puérperas atendidas em 20 Unidades de Saúde (US) do município de Porto Alegre, (RS), Brasil.

O estudo foi aprovado pelo Comitê de Ética em Pesquisa da Universidade Federal de Ciências da Saúde de Porto Alegre (UFCSPA), com parecer de número 545/07, pelo Comitê de Ética da Prefeitura Municipal de Porto Alegre e pelas 20 US participantes. A pesquisa foi conduzida de acordo com a Declaração de Helsinque, revisada em 2008, e registrada no ClinicalTrials.gov, sob o identificador NCT0063545.

De abril a dezembro de 2008, entrevistadores compareceram às US para identificação de gestantes que estivessem no último trimestre de gestação. As gestantes foram informadas sobre os procedimentos do estudo e as que concordaram em participar assinaram o termo de consentimento livre e esclarecido. Nesse momento, as gestantes responderam a um questionário contendo dados referentes a idade, escolaridade, ocupação, paridade e renda familiar total. O peso pré-gestacional foi referido pela gestante, e o peso gestacional foi obtido da carteira de acompanhamento do pré-natal. Obtiveram-se a data provável do parto, o endereço e o contato telefônico da gestante para posterior visita domiciliar. As gestantes diagnosticadas como HIV positivas não foram consideradas elegíveis para o estudo.

Seis e 12 meses após o parto, realizou-se visita domiciliar às mulheres participantes para coleta dos dados antropométricos. As participantes foram pesadas descalças e vestidas com roupas leves com balança digital (Techline ${ }^{\circledR}$ ), com precisão de $100 \mathrm{~g}$. A estatura foi obtida na visita realizada seis meses após o parto, utilizando-se um estadiômetro portátil $\left(\operatorname{Seca}^{\circledR}\right)$, com precisão de $0,1 \mathrm{~cm}$, fixado em parede lisa, com a participante em posição ereta e com os calcanhares encostados na parede.

As mulheres foram avaliadas segundo o Índice de Massa Corpórea (IMC), sendo classificadas como baixo peso $\left(<18,5 \mathrm{~kg} / \mathrm{m}^{2}\right)$, eutrofia $\left(18,5-24,9 \mathrm{~kg} / \mathrm{m}^{2}\right)$, sobrepeso $\left(25-29,9 \mathrm{~kg} / \mathrm{m}^{2}\right)$ e obesidade $\left(\geq 30 \mathrm{~kg} / \mathrm{m}^{2}\right)$. As recomendações adotadas pelo Instituto de Medicina ${ }^{5}$ e pela Organização Mundial da Saúde (OMS) ${ }^{16}$ foram utilizadas para a avaliação durante o período pré-gestacional e 6 e 12 meses após o parto, respectivamente, e a Curva de Atalah ${ }^{17}$ foi utilizada para a avaliação durante a gestação.

O ganho de peso gestacional foi avaliado de acordo com o IMC pré-gestacional ${ }^{5}$ sendo considerado excessivo valor superior a $16,11,5$ e 9,1 kg para as participantes eutróficas, com sobrepeso e obesidade, respectivamente.

A retenção de peso pós-parto foi calculada a partir da subtração do peso pré-gestacional referido pelas gestantes no primeiro momento da coleta de dados e o peso foi aferido após o parto. Para que a avaliação fosse verossímil, as participantes que não apresentaram diferença positiva entre o peso pré-gestacional e o peso após o parto foram excluídas das análises relacionadas à retenção de peso.

O banco de dados foi duplamente digitado no programa estatístico Statistical Package for the Social Sciences (SPSS), versão 11.0 (Statistical Package for Social Sciences Inc., Chicago, Estados Unidos), por 2 estudantes de graduação treinados. A validação dos dados foi feita com o programa Epi Info, versão 6.4 (Centers for Disease Control and Prevention, Atlanta, Estados Unidos) e as análises estatísticas foram realizadas na versão 16.0 do programa SPSS.

As variáveis foram descritas como percentuais ou médias e desvios-padrão. A prevalência de excesso de peso 
(sobrepeso e obesidade) pré-gestacional e 12 meses após o parto foi analisada pelo teste de McNemar. A retenção de peso após o parto foi avaliada pela diferença entre o peso pré-gestacional e o peso aferido 6 e 12 meses após o parto. $\mathrm{O}$ ganho de peso gestacional e a retenção de peso 6 e 12 meses após o parto segundo o IMC pré-gestacional (eutrofia, sobrepeso e obesidade) foram avaliados pela análise de variância (ANOVA) e as comparações múltiplas de Bonferroni. Para avaliar os fatores associados com a retenção de peso 12 meses após o parto, foi utilizada a regressão linear múltipla. Para todos os testes, foi adotado nível de significância de $\mathrm{p}<0,05$.

O valor amostral diferiu em cada análise dependendo da disponibilidade dos dados.

\section{Resultados}

Das 715 gestantes entrevistadas no último trimestre, 633 e 545 foram avaliadas 6 e 12 meses após o parto, respectivamente. Os motivos das perdas durante o seguimento foram recusa em participar do estudo ( $n=62)$, mudança de endereço $(n=100)$ e morte ou doença da mãe e/ou da criança $(n=8)$. As mulheres classificadas como baixo peso pré-gestacional $(n=33)$ e/ou aquelas que reduziram ou mantiveram o peso pré-gestacional 12 meses após o parto $(n=27)$ foram excluídas das análises de ganho de peso gestacional e retenção de peso após o parto.

A maioria das mulheres era branca $(55,3 \%)$, possuía ensino fundamental completo $(52,4 \%)$, não exercia atividade remunerada $(66,7 \%)$ e era multípara $(55,5 \%)$. A média de idade foi de $25,4 \pm 6,6$ anos e $28,5 \%$ eram adolescentes (idade $\leq 20$ anos). A renda familiar mensal de $81,9 \%$ das mulheres foi $\leq 3$ salários mínimos (equivalente a $\mathrm{R} \$ 450,00$ na época da coleta de dados) e a média de renda mensal per capita foi de $\mathrm{R} \$ 320,32 \pm 277,31$.

A distribuição das mulheres de acordo com a classificação do IMC pré-gestacional, gestacional, 6 meses e 12 meses após o parto está descrita na Tabela 1. A prevalência de excesso de peso 12 meses após o parto foi superior ao excesso de peso no período pré-gestacional ( 52,9 versus $36,7 \% ; \mathrm{p}<0,001)$.

A média de ganho de peso gestacional das mulheres estudadas foi de $11,6 \pm 5,7 \mathrm{~kg}$. Considerando-se o IMC pré-gestacional, as mulheres eutróficas $(11,8 \pm 5,5 \mathrm{~kg})$ e com sobrepeso $(12,0 \pm 5,9 \mathrm{~kg})$ apresentaram média de ganho de peso gestacional superior, comparadas àquelas com obesidade $(9,8 \pm 6,3 \mathrm{~kg} ; \mathrm{p}=0,02)$. A prevalência de mulheres com ganho de peso gestacional superior ao valor máximo recomendado de acordo com o IMC pré-gestacional foi de 46,5\% ( $n=59)$ entre aquelas com sobrepeso, $45,9 \%(n=34)$ entre aquelas com obesidade e $17,6 \%(n=61)$ entre as eutróficas.

Seis meses após o parto, $76,4 \%$ das mulheres retiveram peso, e a média foi de $7,4 \pm 6,1 \mathrm{~kg}$. Doze meses após o parto, $72,7 \%$ das mulheres retiveram peso, sendo que $30,7 \%$ retiveram $\geq 10 \mathrm{~kg}$, e a média foi de $8,5 \pm 6,8 \mathrm{~kg}$. Considerando-se o IMC pré-gestacional, a retenção de peso 6 meses $(8,7 \pm 7,0 \mathrm{~kg}$; $\mathrm{p}=0,03)$ e 12 meses $(9,9 \pm 7,7 \mathrm{~kg}, \mathrm{p}=0,03)$ após o parto foi superior nas mulheres com sobrepeso, comparadas às mulheres eutróficas $(6,9 \pm 5,7$ e 7,6 46,2 kg 6 e 12 meses após o parto, respectivamente).

Após a análise de regressão linear, observou-se que a maior retenção de peso 12 meses após o parto foi associada ao maior IMC pré-gestacional, maior ganho de peso gestacional e à gestação na adolescência, como descrito na Tabela 2.

Tabela 2. Fatores associados à retenção de peso 12 meses após o parto

\begin{tabular}{lcc}
\hline & Coeficiente de regressão $(\beta)$ & Valor $p$ \\
\hline Valor de IMC pré-gestacional & 0,23 & $<0,001$ \\
Ganho de peso gestacional & 0,33 & $<0,001$ \\
Idade $\leq 20$ anos & 0,23 & $<0,001$ \\
Tempo de estudo & $-0,06$ & 0,25 \\
Renda per capita & $-0,03$ & 0,58 \\
Paridade & $-0,07$ & 0,19 \\
Tempo de aleitamento materno exclusivo & $-0,20$ & 0,32 \\
\hline
\end{tabular}

IMC: índice de massa corpórea.

Tabela 1. Distribuição da amostra de acordo com o índice de massa corpórea pré-gestacional, gestacional, 6 e 12 meses após o parto

\begin{tabular}{|c|c|c|c|c|c|c|c|c|}
\hline & \multicolumn{2}{|c|}{$\begin{array}{l}\text { Pré-gestacional } \\
(\mathrm{n}=624)\end{array}$} & \multicolumn{2}{|c|}{$\begin{array}{c}\text { Gestacional* } \\
(n=544)\end{array}$} & \multicolumn{2}{|c|}{$\begin{array}{l}6 \text { meses após o parto } \\
(n=607)\end{array}$} & \multicolumn{2}{|c|}{$\begin{array}{c}12 \text { meses após o parto } \\
(n=505)\end{array}$} \\
\hline Baixo peso & 5,3 & 33 & 10,3 & 56 & 1,6 & 10 & 3,0 & 15 \\
\hline Sobrepeso & 22,1 & 138 & 27,8 & 151 & 27,8 & 169 & 28,7 & 145 \\
\hline Obesidade & 14,6 & 91 & 23,5 & 128 & 24,2 & 147 & 24,2 & 122 \\
\hline
\end{tabular}

*A avaliação do índice de massa corpórea gestacional foi realizada no último trimestre da gestação. 


\section{Discussão}

Os resultados do presente estudo mostraram que aproximadamente $50 \%$ das mulheres com sobrepeso ou obesidade apresentaram ganho de peso gestacional superior ao recomendado pelo Instituto de Medicina ${ }^{5}$ e que mais de $70 \%$ retiveram peso até 12 meses após o parto — sendo que $30 \%$ retiveram $10 \mathrm{~kg}$ ou mais, aumentando, assim, a prevalência de mulheres com excesso de peso após a gestação. Os fatores associados à retenção de peso foram IMC pré-gestacional, ganho de peso gestacional e idade.

O maior IMC pré-gestacional foi fator de risco para a retenção de peso após o parto entre as mulheres estudadas, consolidando evidências que mostram que o risco de reter peso após o parto aumenta entre mulheres que iniciam a gestação com sobrepeso ou obesidade ${ }^{15,18-20}$. Porém, houve ainda redução do percentual de mulheres eutróficas no período após o parto, o que pode ser explicado pela provável insuficiência de vigilância no controle do ganho de peso gestacional entre essas mulheres, uma vez que elas não fazem parte do grupo de risco no início da gestação. Mulheres que extrapolam as recomendações para ganho de peso durante a gestação se expõem a maiores riscos de desenvolverem excesso de peso ou obesidade tanto no curto quanto no longo prazo ${ }^{12,21-23}$.

Assim, o ganho de peso gestacional excessivo mostrou-se preditor para maior retenção de peso após o parto, corroborando outros estudos ${ }^{1,21,23,24}$. Estudo prospectivo realizado para avaliar o efeito do ganho de peso gestacional em longo prazo mostrou que o ganho de peso gestacional excessivo aumentou o risco de desenvolver sobrepeso ou obesidade em até duas décadas após a gestação ${ }^{22}$. Mannan et al. ${ }^{21}$, por meio da avaliação de uma série de meta-análises para investigar a associação de ganho de peso gestacional com a retenção de peso em até 21 anos após o parto, encontraram que o ganho de peso gestacional excessivo tem maior influência na retenção de peso após o parto do que na situação oposta de pouco ganho de peso e manutenção dessa condição no pós-parto. Essa situação de facilitação para aumento nas reservas de gordura é promovida pelos mecanismos fisiológicos que ocorrem na gestação como o maior acúmulo de triglicerídeos para garantir reserva imediata de energia para o feto em períodos de jejum prolongado ${ }^{25}$.

Estudo recente mostrou que $50 \%$ das mulheres apresentavam excesso de peso durante a gestação e, quando questionadas sobre seu estado nutricional, a maioria considerava seu peso normal e apenas $24 \%$ identificou-se com excesso de peso ${ }^{26}$. É possível, portanto, que o aumento da prevalência da obesidade esteja mudando a percepção do que é considerado adequado pela população. Outro aspecto interessante do estudo foi que as gestantes consideravam a ingestão de alimentos orgânicos, sucos de frutas e não se alimentar após as 20 horas comportamentos adequados para o controle do ganho de peso ${ }^{26}$.

Outro fator de risco para retenção de peso após o parto foi ser adolescente, isto é, mulheres com idade menor ou igual a 20 anos, corroborando outros 2 estudos prospectivos realizados com mulheres brasileiras ${ }^{6}$ e americanas ${ }^{12}$. Gigante et al. ${ }^{27}$, em estudo realizado com adolescentes de uma coorte de nascimento de base populacional do sul do Brasil, mostraram que a gestação durante a adolescência foi associada ao aumento da prevalência de obesidade na vida adulta. Informações específicas e atuais sobre gestação entre adolescentes ainda se mostram limitadas, sendo necessários mais estudos nessa área.

O presente estudo não encontrou associação entre o aleitamento materno exclusivo e retenção de peso 12 meses após o parto. Estudos mostram resultados controversos quanto à influência da amamentação na retenção de peso após o parto ${ }^{1,20,28-31}$ e sugerem que a amamentação por si só não previne a retenção de peso após o parto ${ }^{24}$, mas pode ser um dos fatores que contribui para a perda de peso pós-parto ${ }^{24,32}$.

Quanto às limitações deste estudo, destaca-se que o peso pré-gestacional foi referido pelas participantes, de tal forma que o valor pode ter sido subestimado ${ }^{6,33}$. Entretanto, um estudo mostrou que a avaliação do estado nutricional por meio do peso pré-gestacional referido pode ser um indicador confiável quando há aferição precisa da estatura - como realizado no presente estudo — devido à redução no erro no cálculo do IMC ${ }^{34}$. Outra limitação diz repeito à extrapolação dos resultados para outros grupos de melhor condição socioeconômica, já que os dados deste estudo foram obtidos de grupo populacional de menor poder aquisitivo.

Intervenções nutricionais durante a gestação podem ajudar na prevenção do ganho de peso gestacional excessivo e na redução da retenção de peso após o parto ${ }^{35}$. Estudo em nosso meio mostrou que a intervenção nutricional durante a gestação reduziu a velocidade de ganho de peso para gestantes com excesso de peso, porém não foi efetiva para prevenir o ganho de peso excessivo entre eutróficas. Além disso, os autores do mesmo estudo enfatizaram que a intervenção deve iniciar antes da $20^{a}$ semana gestacional $^{36}$. Conclui-se, desta forma, que é necessária a adequada assistência pré-natal para minimizar os efeitos adversos do ganho de peso excessivo durante a gestação na saúde da mulher.

\section{Agradecimentos}

Ao Ministério da Saúde (termo de portaria: 577/200) e à Fundação de Amparo à Pesquisa do Rio Grande do Sul (PPSUS/2006/1537-7). 
1. Baker JL, Gamborg M, Heitmann BL, Lissner L, Sørensen TI, Rasmussen KM. Breastfeeding reduces postpartum weight retention. Am J Clin Nutr. 2008;88(6):1543-51.

2. Ostbye T, Krause KM, Lovelady CA, Morey MC, Bastian LA, Peterson $\mathrm{BL}$, et al. Active mothers postpartum: a randomized controlled weight-loss intervention trial. Am J Prev Med. 2009;37(3):173-80.

3. Lipsky LM, Strawderman MS, Olson CM. Maternal weight change between 1 and 2 years postpartum: the importance of 1 year weight retention. Obesity (Silver Spring). 2012;20(7): 1496-502.

4. Frischknecht F, Brühwiler H, Raio L, Lüscher KP. Changes in pre-pregnancy weight and weight gain during pregnancy: retrospective comparison between 1986 and 2004. Swiss Med Wkly. 2009;139(3-4):52-5.

5. National Academy of Science. Institute of Medicine. Weight gain during pregnancy. Reexaming the guidelines. Washington (DC): National Academy Press; 2009.

6. Kac G, Benicio MH, Velásquez-Meléndez G, Valente JG. Nine months postpartum weight retention predictors for Brazilian women. Public Health Nutr. 2004;7(5):62 1-8.

7. Nogueira Al, Carreiro MP. Obesidade e gravidez. Rev Med Minas Gerais. 2013;23(1):88-98.

8. Santos EMF, Amorim LP, Costa OLN, Oliveira N, Guimarães AC. [Profile of gestational and metabolic risk in the prenatal care service of a public maternity in the Brazilian Northeast]. Rev Bras Ginecol Obstet. 2012;34(3):102-6.

9. Marano D, Gama SGN, Pereira APE, Souza Junior PRB. Adequacy of weight gain in pregnant women from two municipalities of Rio de Janeiro state (RJ), Brazil, 2008. Rev Bras Ginecol Obstet. 2012;34(8):386-93.Portuguese.

10. Nucci LB, Duncan BB, Mengue SS, Branchtein L, Schmidt MI, Fleck $E T$. Assessment of weight gain during pregnancy in general prenatal care services in Brazil. Cad Saúde Pública. 2001;17(6):1367-74.

11. Viswanathan M, Siega-Riz AM, Moos MK, Deierlein A, Mumford $S$, Knaack J, et al. Outcomes of maternal weight gain. Rockville, MD: Agency for Healthcare Research and Quality; 2008. (Evidence Report/Technology Assessment, 168).

12. Olson CM, Strawderman MS, Hinton PS, Pearson TA. Gestational weight gain and postpartum behaviors associated with weight change from early pregnancy to 1 y postpartum. Int J Obes Relat Metab Disord. 2003;27(1):117-27.

13. Amorim AR, Rössner $S$, Neovius $M$, Lorenço PM, Linné $Y$. Does excess pregnancy weight gain constitute a major risk for increasing long-term BMI? Obesity (Silver Spring). 2007;15(5): 1278-86.

14. Rebelo F, Castro MBT, Dutra CL, Schlussel MM, Kac G. Fatores associados à retenção de peso pós-parto em uma coorte de mulheres, 2005-2007. Rev Bras Saúde Mater Infant. 2010;10(2):219-27.

15. Castro MBT, Kac G, Sichieri R. Determinantes nutricionais e sóciodemográficos da variação de peso no pós-parto: uma revisão da literatura. Rev Bras Saúde Mater Infant. 2009;9(2): 125-37.

16. World Health Organization. Obesity: preventing and managing the global epidemic. Report of the WHO Consultation on Obesity. Geneva: WHO; 1998.

17. Atalah Samur E, Castillo LC, Castro Santoro R, Aldea PA. Propuesta de un nuevo estándar de evaluación nutricional en embarazadas. Rev Méd Chile. 1997;125(12): 1429-36.

18. Gore SA, Brown DM, West DS. The role of postpartum weight retention in obesity among women: a review of the evidence. Ann Behav Med. 2003;26(2): 149-59.
19. Lacerda EMA, Leal MC. Fatores associados com a retenção e o ganho de peso pós-parto: uma revisão sistemática. Rev Bras Epidemiol. 2004;7(2):187-200.

20. Sichieri R, Field AE, Rich-Edwards J, Willett WC. Prospective assessment of exclusive breastfeeding in relation to weight change in women. Int J Obes Relat Metab Disord. 2003;27(7):815-20.

21. Mannan M, Doi SA, Mamun AA. Association between weight gain during pregnancy and postpartum weight retention and obesity: a bias-adjusted meta-analysis. Nutr Rev. 2013;71(6):343-52.

22. Vesco KK, Dietz PM, Rizzo J, Stevens VJ, Perrin NA, Bachman DJ, et al. Excessive gestational weight gain and postpartum weight retention among obese woman. Obstet Gynecol. 2009; 1 14(5):1069-75.

23. Lederman SA. Influence of lactation on body weight regulation. Nutr Rev. 2004;62(7 Pt 2):S1 12-9.

24. Mamun AA, Kinarivala M, O'Callaghan M, Williams GM, Najman JM, Callaway LK. Associations of excess weight gain during pregnancy with long-term maternal overweight: evidence from 21 y postpartum follow-up. Am J Clin Nutr. 2010;91 (5):1336-41.

25. National Academy of Science. Institute of Medicine. Nutrition during pregnancy: Part I: weight gain, Part II: nutrient supplements. Washington (DC): National Academy Press; 1990.

26. Shub A, Huning EY, Campbell KJ, McCarthy EA. Pregnant women's knowledge of weight, weight gain, complications of obesity and weight management strategies in pregnancy. BMC Res Notes. 2013;6:278.

27. Gigante DP, Rasmussen KM, Victora CG. Pregnancy increases $\mathrm{BMI}$ in adolescents of a population-based birth cohort. J Nutr. 2005; 135(1):74-80

28. Kac G, Benício MH, Velásquez-Meléndez G, Valente JG, Struchiner CJ. Breastfeeding and postpartum weight retention in a cohort of Brazilian women. Am J Clin Nutr. 2004;79(3):487-93.

29. Coitinho DC, Sichieri R, Benício MH. Obesity and weight change related to parity and breast-feeding among parous women in Brazil. Public Health Nutr. $2001 ; 4(4): 865-70$.

30. Krause KM, Lovelady CA, Peterson BL, Chowdhury N, Østbye T. Effect of breast-feeding on weight retention at 3 and 6 months postpartum: data from the North Carolina WIC Programme. Public Health Nutr. 2010;13(2):2019-26.

31. Biesmans K, Franck E, Ceulemans C, Jacquemyn Y, Van Bogaert P. Weight during the postpartum period: what can health care workers do? Matern Child Health J. 2013;17(6):996-1004.

32. Onyango AW, Nommsen-Rivers L, Siyam A, Borghi E, Onis M, Garza $\mathrm{C}$, et al. Post-partum weight change patterns in the WHO Multicentre Growth Reference Study. Matern Child Nutr. 201 1;7(3):228-40.

33. Pedersen P, Baker JL, Henriksen TB, Lissner L, Heitmann BL, Sørensen $\mathrm{TI}$, et al. Influence of psychosocial factors on postpartum weight retention. Obesity (Silver Spring). $2011 ; 19(3): 639-46$.

34. Mardones F, García-Huidobro T, Ralph C, Farías M, Domínguez A, Rojas I, et al. Influencia combinada del índice de masa corporal pregestacional y de la ganancia de peso en el embarazo sobre el crecimiento fetal. Rev Méd Chile. 2011;139(6):710-6.

35. Brawarsky P, Stotland NE, Jackson RA, Fuentes-Afflick E, Escobar GJ, Rubashkin N, et al. Pre-pregnancy and pregnancy related factors and the risk of excessive or inadequate gestational weight gain. Int J Gynaecol Obstet. 2005;91 (2):125-31.

36. Vitolo MR, Bueno MSF, Gama CM. [Impact of a dietary counseling program on the gain weight speed of pregnant women attended in a primary care service]. Rev Bras Ginecol Obstet. 201 1;33(1): 13-9. 